\title{
Decreased Neuroplasticity May Play a Role in Irritable Bowel Syndrome: Implication From the Comorbidity of Depression and Irritable Bowel Syndrome
}

TO THE EDITOR: It is with great interest that we read the paper entitled "Post-traumatic Stress Disorder Is Associated With Irritable Bowel Syndrome in African Americans" by Iorio et al. ${ }^{1}$ The authors investigated the potential association between irritable bowel syndrome (IBS) and post-traumatic stress disorder (PTSD) in an urban African American (AA) population. The results demonstrate that those with IBS were much more likely to suffer from PTSD; PTSD is independently associated with depression and anxiety; and the majority of individuals with IBS suffered from depression, and anxiety was the second most common in IBS patients. Then it is concluded that PTSD is independently associated with IBS. The authors also emphasized the relation between hypothalamic-pituitary-adrenal (HPA) hormones, such as corticotropin-releasing hormone, adrenocorticotropic hormone and cortisol, and IBS. Although the authors pointed out that this study specifically addressed AA and could not be extrapolated to other races, we believe that it enlightened the relation between stress and IBS in the whole population.

Neuroplasticity is the ability of the nervous system to respond to intrinsic or extrinsic stimuli by reorganizing its structure, function and connections. ${ }^{2}$ The nervous system monitors and coordinates internal organ function, therefore we have proposed that neuroplasticity may also be associated with the pathogenesis of other diseases besides neuropsychiatric diseases. ${ }^{3}$ IBS has been generally considered to be caused by alterations in the brain-gut axis. ${ }^{4}$ Thus neuroplasticity may be related to the pathogenesis of IBS.

Neuroplasticity is disrupted in mood disorders and depression is a disorder of decreased neuroplasticity. ${ }^{3,5}$ It is widely accepted that stress triggers the activation of the HPA axis and causes the brain to be exposed to corticosteroids, affects neurobehavioral functions with a strong down-regulation of hippo- campal neurogenesis, and is a pivotal risk factor for depression. Brain-derived neurotrophic factor (BDNF) is a critical cytokine in neuronal survival, morphogenesis, and plasticity. BDNF expression is regulated by stress-responsive corticosteroids, and increased glucocorticoid exposure induces a reduction in BDNF level. Most of the circulating BDNF is produced in the brain and passes through the blood-brain barrier. Serum BDNF level is believed as a biomarker for depression. A meta-analysis showed a significant reduction of serum BDNF level in depression. ${ }^{3}$ Shortened and reduced complexity of dendritic trees have been found in the hippocampus and the prefrontal cortex in depression. Antidepressant treatment increases the expression of BDNF and can enhance neuroplasticity. ${ }^{5}$

Besides depression, some other psychological disorders, which IBS is commonly comorbid with, such as anxiety, are also related to decreased neuroplasticity. ${ }^{6}$ Thus it is suggested that decreased neuroplasticity induces the comorbidity of depression/anxiety and IBS, and IBS is closely associated with decreased neuroplasticity.

There is much evidence for the association of IBS and decreased neuroplasticity. As suggested by the reviewing paper, besides depression, IBS is also closely associated with stress, which may decrease neuroplasticity. ${ }^{1,3}$ The levels of both glucocorticoid and BDNF in IBS, which are critical factors in neuroplasticity, are similar to those in depression. For example, cortisol was elevated in all IBS subgroups (diarrhea predominant, constipated, and alternators) in humans, ${ }^{7}$ and IBS rats showed depressionand anxiety-like behaviors associated with decreased BDNF expression in the hippocampus after chronic acute combining stress. ${ }^{8}$ Physiological analysis of IBS patients indicated decreased gray matter density (thickness) in widespread areas of the brain, such as the medial prefrontal cortex, ventrolateral prefrontal cor- 
tex, and left dorsolateral prefrontal cortex. ${ }^{9}$ A research result shows that neural degeneration in the myenteric plexus connected with inflammatory changes may play a role in the pathogenesis of IBS. ${ }^{10}$

Thus, to our point, decreased neuroplasticity may cause IBS. This is why IBS is commonly comorbid with depression. And increased neuroplasticity may protect against IBS. This could explain the use of antidepressant for the treatment of IBS since antidepressant treatment can increase neuroplasticity. ${ }^{1,5}$ As stated above, decreased neuroplasticity may also cause anxiety. Thus anxiety is also common in IBS.

Zhihua Zheng ${ }^{1}$ and Hongmei Tang ${ }^{2}$ ${ }^{1}$ Guangdong Province Pharmaceutical Association, Guangzhou, China; and ${ }^{2}$ The First Affiliated Hospital of Guangzhou University of Traditional Chinese Medicine, Guangzhou, China

1. Iorio N, Makipour K, Palit A, Friedenberg FK. Post-traumatic stress disorder is associated with irritable bowel syndrome in African Americans. J Neurogastroenterol Motil 2014;20:523-530.

2. Cramer SC, Sur M, Dobkin BH, et al. Harnessing neuroplasticity for clinical applications. Brain 2011;134(Pt 6):1591-1609.

3. Zheng Z, Zeng Y, Wu J. Increased neuroplasticity may protect against cardiovascular disease. Int J Neurosci 2013;123:599-608.

4. Fichna J, Storr MA. Brain-Gut Interactions in IBS. Front Pharmacol 2012;3:127.

5. Pittenger C, Duman RS. Stress, depression, and neuroplasticity: a convergence of mechanisms. Neuropsychopharmacology 2008;33:88-109.

6. Domingos da Silveira da Luz AC, Pereira Dias G, do Nascimento Bevilaqua MC, et al. Translational findings on brain-derived neurotrophic factor and anxiety: contributions from basic research to clinical practice. Neuropsychobiology 2013;68:129-138.

7. Dinan TG, Quigley EM, Ahmed SM, et al. Hypothalamic-pituitary-gut axis dysregulation in irritable bowel syndrome: plasma cytokines as a potential biomarker? Gastroenterology 2006;130:304-311.

8. Yu Y, Wu S, Li J, et al. The effect of curcumin on the brain-gut axis in rat model of irritable bowel syndrome: involvement of 5-HT-dependent signaling. Metab Brain Dis 2015;30:47-55.

9. Seminowicz DA, Labus JS, Bueller JA, et al. Regional gray matter density changes in brains of patients with irritable bowel syndrome. Gastroenterology 2010;139:48-57, e2.

10. Törnblom H, Lindberg G, Nyberg B, Veress B. Full-thickness biopsy of the jejunum reveals inflammation and enteric neuropathy in irritable bowel syndrome. Gastroenterology 2002;123:1972-1979.

\section{Conflicts of interest: None.}

